\title{
Avant-propos et remerciements
}

Cet ouvrage s'appuie sur le vécu d'analyse de la sûreté des réacteurs nucléaires électrogènes, de type REP, et sur une quinzaine d'années d'enseignement de l'auteur sur le sujet. II reprend, en grande partie, les différents supports de cours, travaux dirigés et travaux pratiques sur simulateur de fonctionnement accidentel, du cours de Génie Atomique de I'Institut National des Sciences et Techniques Nucléaires.

Il a pour objectif de présenter le comportement du réacteur en situations accidentelles en s'appuyant sur les bases neutroniques et thermohydrauliques, ainsi que la description fonctionnelle de la chaudière. Sa valeur ajoutée, par rapport aux autres ouvrages de la collection, est d'aborder la dimension systémique du fonctionnement d'un réacteur nucléaire, caractérisée par de nombreux couplages entre phénomènes physiques et des interactions dynamiques entre les sous-parties du système. Une telle approche est indispensable pour comprendre, interpréter et prédire le comportement du système réacteur, compétences requises pour l'exercice des métiers de la conception, de l'exploitation et bien sûr de l'analyse de sûreté de ce type d'installation.

L'ouvrage se détache en deux parties, l'une présentant les principales familles d'accidents susceptibles d'affecter les trois fonctions de sûreté, l'autre, l'accident de Three Mile Island, accident de référence pour les REP, et ses enseignements en termes d'optimisation de la conduite post-accidentelle pour éviter la fusion du cœur, et garantir le confinement. La conclusion tente de synthétiser les conditions de la maîtrise des situations accidentelles sur ce type $d^{\prime}$ installation.

Chaque chapitre fait l'objet d'un ou deux problème(s), exploitant un modèle REP ponctuel, pour permettre d'analyser qualitativement les transitoires étudiés et de caractériser quantitativement les états d'équilibre dynamique sur lesquels le réacteur converge, du fait de sa grande stabilité.

De nombreuses annexes apportent des compléments d'informations, utiles à tout ingénieur s'intéressant à ce sujet, comme par exemple les enseignements des autres grands accidents nucléaires, les démarches d'analyse de sûreté déterministe ou probabiliste ou encore la composante humaine et organisationnelle du système sociotechnique.

L'auteur voudrait remercier Joseph Safieh pour le témoignage de sa confiance, ainsi que les personnes qui ont contribué à la réalisation de cet ouvrage, en particulier les membres de l'équipe pédagogique et les élèves ingénieurs du Génie Atomique de l'INSTN.

Il tient cependant à remercier tout particulièrement Olivier Hascoët et ses collègues de la division Physique des Réacteurs d'EDF/SEPTEN qui ont accepté de relire collégialement I'intégralité du document et ont fait de nombreuses suggestions pour en garantir la lisibilité et la rigueur technique.

Toutes les erreurs et omissions restent, bien évidemment, de la seule responsabilité de I'auteur. 
7 KVSDJHIQQMQWRQDCIOHVEQN 